\title{
ON INTEGRAL COHOMOLOGY OF CERTAIN ORBIFOLDS
}

\author{
ANTHONY BAHRI, DIETRICH NOTBOHM, SOUMEN SARKAR, AND JONGBAEK SONG
}

\begin{abstract}
The CW structure of certain spaces, such as effective orbifolds, can be too complicated for computational purposes. In this paper we use the concept of q-CW complex structure on an orbifold, to detect torsion in its integral cohomology. The main result can be applied to well known classes of orbifolds or algebraic varieties having orbifold singularities, such as toric orbifolds, simplicial toric varieties, torus orbifolds and weighted Grassmannians.
\end{abstract}

\section{INTRODUCTION}

Recent years have seen the development of an abundance of specialized tools for the study of orbifolds. A variety of cohomology theories have been crafted specifically to investigate particular classes of orbifolds arising in complex geometry, algebraic geometry, symplectic geometry and string topology. Among these are: the de Rham cohomology of orbifolds [ALR07, Chapter 2], orbifold Dolbeault cohomology Bai54, Bai56, Sat56, Bott-Chern orbifold cohomology Ang13, orbifold quantum cohomology [CLCT09, Section 2.2.5], intersection homology [GM80] and the ChenRuan orbifold cohomology ring [CR04].

These theories do yield information about the ordinary singular cohomology of orbifolds but the coefficients tend to be real, complex or rational, as appropriate. In general, the singular integral cohomology ring of a given orbifold remains sometimes intractable object, difficult to compute.

Among orbifolds for which the integral cohomology ring does succumb to the traditional methods of algebraic topology, are those having cohomology which can be shown to be concentrated in even degree. The prime example is that of weighted projective spaces. Additively, their integral cohomology agrees with that for ordinary projective spaces but the ring structure is complicated by an abundance of divisibility arising from the weights, [Kaw73, AA97, BSS17, Section 5.1]. Included also in this class of "nice" orbifolds are: weighted Grassmannians CR02, AM15, certain singular toric varieties, toric orbifold 1 , and orbifolds which arise as complex vector bundles over spaces with even cohomology.

With this in mind, the determination of verifiable sufficient conditions on an orbifold, which ensure that the integral cohomology is concentrated in even degrees, becomes a natural question. Motivated by Kawasaki's computation for weighted projective spaces, the approach taken here begins by identifying classes of orbifolds which can be constructed by a sequence of canonical cofibrations, in a manner not unlike that for $\mathrm{CW}$ complexes. We employ ideas originated by Goresky in Gor78]

Date: November 7, 2017.

2010 Mathematics Subject Classification. 57R18, 57R19, 14M15.

Key words and phrases. orbifold, CW-complex, group action, homology group, cohomology ring, Grassmannian.

${ }^{1}$ In the literature, these are also called quasi-toric orbifolds. 
and developed in a toric context by Poddar and Sarkar in PS10. Confounding the process however, is the ineluctable need to replace ordinary cells with "q-cells", the quotient of a disc by the action of a finite group. The task reduces then to keeping at bay the intrusion of torsion into odd cohomological degrees.

The basic structure of a q-CW complex is reviewed and developed further in Section 2. We note in Proposition 2.4 that a $\mathbf{q}-\mathrm{CW}$ complex is homotopic to a simplicial complex. In order to compute the integral cohomology of a $\mathbf{q}-\mathrm{CW}$ complex inductively, using a specific sequence of cofibrations, we introduce the notion of a building sequence $\left\{\left(Y_{i}, 0_{i}\right)\right\}_{i=1}^{\ell}$. Here, $Y_{i}$ is obtained from $Y_{i-1}$ by attaching a q-cell of the form $e^{k_{i}} / G_{i}$, where $e^{k_{i}}$ is an open disc and $G_{i}$ is a finite group acting linearly. The image of the origin $0_{i}$ in the $\mathbf{q}$-cell $e^{k_{i}} / G_{i}$ plays an important role.

The results in the following sections are anchored in the observation that when a finite group $G$ acts linearly on a closed disc $\bar{D}^{n}$, the torsion which can arise in $H_{*}\left(S^{n-1} / G ; \mathbb{Z}\right)$ depends on $|G|$ only. In particular, we conclude that if $p$ is co-prime to all the primes dividing $|G|$, then $H_{*}\left(S^{n-1} / G ; \mathbb{Z}\right)$ has no $p$-torsion.

Section 3 contains the main theorem about $\mathbf{q}-\mathrm{CW}$ complexes.

Theorem 1.1. Let $X$ be a q- $C W$ complex with no odd dimensional $\mathbf{q}$-cells and $p$ a prime number. If $\left\{\left(Y_{i}, 0_{i}\right)\right\}_{i=1}^{\ell}$ is a building sequence such that $\operatorname{gcd}\left\{p,\left|G_{i}\right|\right\}=1$ for all $i$ with $e^{2 k_{i}} / G_{i}=Y_{i} \backslash Y_{i-1}$, then $H_{*}(X ; \mathbb{Z})$ has no $p$-torsion and $H_{\text {odd }}\left(X ; \mathbb{Z}_{p}\right)$ is trivial.

Successive applications of this theorem yield its important theorem.

Theorem 1.2. Let $X$ be a $\mathbf{q}-C W$ complex with no odd dimensional $\mathbf{q}-$ cells. If for each prime $p$ there is a building sequence $\left\{\left(Y_{i}, 0_{i}\right)\right\}_{i=1}^{\ell}$ such that $\operatorname{gcd}\left\{p,\left|G_{i}\right|\right\}=1$ for all $i$ with $e^{2 k_{i}} / G_{i}=Y_{i} \backslash Y_{i-1}$, then $H_{*}(X ; \mathbb{Z})$ has no torsion and $H_{\text {odd }}(X ; \mathbb{Z})$ is trivial.

Applications and examples are given in Section 4 The topological construction of a toric orbifold $X(Q, \lambda)$, arising from a simple polytope $Q$ and an $\mathcal{R}$-characteristic pair $(Q, \lambda)$, is reviewed in Subsection 4.1. Then, we re-visit the notion of a retraction sequence of triples, $\left\{\left(B_{k}, E_{k}, v_{k}\right)\right\}_{k=1}$, for a simple polytope $Q$, which was introduced in BSS17. Each space $B_{k}$ is a polytopal complex obtained by removing successively, carefully chosen vertices, beginning with $Q$ itself. Each $E_{k}$ is a particular face in $B_{k}$ and $v_{k}$ is a special vertex in $E_{k}$ called a free vertex. Then Proposition 4.4 provides the means by which we can use a q-CW structure to study toric orbifolds. So, a toric orbifold which satisfies the hypothesis of Theorem 1.2 has cohomology which is concentrated in even degrees. Moreover, from the point of view of retraction sequences, the orders $\left|G_{i}\right|$ appearing in Theorem 1.2 , are computable explicitly from the $\mathcal{R}$-characteristic data $(Q, \lambda)$, which may be denoted as $g_{E_{i}}\left(v_{i}\right)$. This result appears in Theorem 4.6. An example is given next which proves that the gcd condition in Theorem 4.6 is weaker than the hypothesis of BSS17, Theorem 1.1], the main result in that paper about the integral cohomology of toric orbifolds.

Our attention turns in Subsection 4.2 to the study of certain projective toric varieties. Following a brief review of the basic construction of a toric variety $X_{\Sigma}$ from a fan $\Sigma$, we note that a simplicial polytopal fan arises as the normal fan of a simple polytope $Q$. Moreover, the fan determines an $\mathcal{R}$-characteristic pair $(Q, \lambda)$. 
In this case, we have

$$
X(Q, \lambda) \cong X_{\Sigma}
$$

as orbifolds with torus action. The results of Section 3 are applied then to this class of toric varieties. When the results are combined with [BSS17, Theorem 5.4], we get a complete description of the integral cohomology ring under conditions weaker than those of [BSS17, Theorem 1.2].

In Subsection 4.3, retraction sequence techniques are shown, under certain conditions, to apply equally well to a generalization of a toric orbifold called a torus orbifold, introduced by Hattori and Masuda HM03. Here, the simple polytope $Q$ is replaced by a manifold with corners $P$ which has additional properties. As observed by Masuda and Panov [MP06, Section 4.2], they too can be constructed by an $\mathcal{R}$-characteristic pair $(P, \lambda)$. This allows us to obtain a result for torus orbifolds similar to Theorem 4.6.

A discussion of weighted Grassmannians follows in Subsection 4.4. Abe and Matsumura [AM15, Section 2] show that the Schubert cell structure, which is indexed by Young diagrams, determines a q-cell structure on weighted Grassmannians. This allows for an application of Theorem 1.1 at the end of this subsection.

The paper concludes with a discussion of $\mathbf{q}-\mathrm{CW}$ complexes which may have odd dimensional q-cells. The main theorem gives a condition on a prime $p$ and a building sequence $\left\{\left(Y_{i}, 0_{i}\right)\right\}_{i=1}^{\ell}$ for a $\mathbf{q}-\mathrm{CW}$ complex $X$ which ensures that $H_{*}(X ; \mathbb{Z})$ has no $p$-torsion. Also a condition of having $p$-torsion in $H_{*}(X ; \mathbb{Z})$ is given.

Acknowledgements: This work was supported in part by grants 210386 and 426160 from Simons Foundation. The fourth author was partially supported by Basic Science Research Program through the National Research Foundation of Korea (NRF) funded by the Ministry of Science, ICT \& Future Planning (No. 2016R1A2B4010823)

\section{2. q-CW COMPLEXES}

In this section, we introduce the notion of $\mathbf{q}-\mathrm{CW}$ complex structure in which the analogue of an open cell is the quotient of an open disk by an action of a finite group. The construction mirrors the construction of ordinary $\mathrm{CW}$ complexes given, for example, in Hatcher Hat02. We note that q-CW complex structures were used to compute the rational homology of certain singular spaces having torus symmetries in [PS10].

Definition 2.1. Let $G$ be a finite group acting linearly on a closed $n$-dimensional disc $\bar{D}^{n}$ centered at the origin. Such an action preserves $\partial \bar{D}^{n}=S^{n-1}$. We call the quotient $\bar{D}^{n} / G$ an $n$-dimensional $\mathbf{q}$-disc. If $\bar{e}^{n}$ is $G$-equivariantly homeomorphic to $\bar{D}^{n}$, we call $e^{n} / G$ a $\mathbf{q}-$ cell. By abuse of notation, we shall denote the boundary of $\bar{e}^{n}$ by $S^{n-1}$ without confusion.

A q-CW complex is constructed inductively as follows. Start with a discrete set $X_{0}$, where points are regarded as 0-dimensional q-cells. Inductively, form the $n$-dimensional q-skeleton $X_{n}$ from $X_{n-1}$ by attaching finitely many $n$-dimensional q-cells $\left\{e^{n} / G_{\alpha}\right\}$ via continuous maps $\left\{\phi_{\alpha}\right\}$ where $\phi_{\alpha}: S^{n-1} / G_{\alpha} \rightarrow X_{n-1}$. This means that $X_{n}$ is the quotient space of the disjoint union $X_{n-1} \bigsqcup_{\alpha}\left\{\bar{e}^{n} / G_{\alpha}\right\}$ of $X_{n-1}$ with a finite collection of $n$-dimensional q-disks $\bar{e}^{n} / G_{\alpha}$ under the identification $x \sim \phi_{\alpha}(x)$ for $x \in S^{n-1} / G_{\alpha}$. 
If $X=X_{n}$ for some finite $n$, we call $X$ a finite $\mathbf{q}-C W$ complex of dimension $n$. The topology of $X$ is the quotient topology built inductively. We say $Y$ is a q-CW subcomplex of $X$, if $Y$ is a q-CW complex and $Y$ is a subset of $X$.

Definition 2.2. (1) Let $0_{i} \in X$ denote the image of origin in the q-cell $e^{k_{i}} / G_{i}$. We call $0_{i}$ a special point corresponding to the $i$-th $\mathbf{q}$-cell. We may also refer to $0_{i}$ as a special point of $X$.

(2) Let $Y$ be a q-CW subcomplex of a q-CW complex $X$. Then a special point $0_{i} \in Y$ is called free in $Y$ if there is a neighborhood of $0_{i}$ in $Y$ homeomorphic to $e^{k} / G_{i}$ for some $k \in \mathbb{Z}_{\geq 0}$ and finite group $G_{i}$.

Remark 2.3. (1) Every finite q-CW complex has a free special point. A finite $\mathbf{q}-\mathrm{CW}$ complex can be obtained by attaching one $\mathbf{q}$-cell at each time. Attaching q-cells need not be in increasing dimension.

(2) If $0_{i}$ is a free special point of a $\mathbf{q}-\mathrm{CW}$ complex $Y$, then $Y$ can be obtained from a $\mathbf{q}-\mathrm{CW}$ subcomplex $Y^{\prime}$ by attaching a $\mathbf{q}$-cell $\bar{e}^{k_{i}} / G_{i}$. In this case, the natural deformation retract from $\left(\bar{e}^{k_{i}} \backslash\left\{0_{i}\right\}\right) / G_{i}$ to $\partial \bar{e}^{k_{i}} / G_{i}$ induces a deformation retract from $Y \backslash\left\{0_{i}\right\}$ to $Y^{\prime}$.

(3) Let $S_{\frac{1}{2}}^{k-1}=\left\{x \in e^{k} \cong D^{k}|| x \mid=\frac{1}{2}\right\}$. Then $S_{\frac{1}{2}}^{k-1} / G_{i} \cong S^{k-1} / G_{i}$ and the previous remark implies that the following is a cofibration:

$$
S^{k-1} / G_{i} \stackrel{\phi_{i}}{\longrightarrow} Y^{\prime} \longrightarrow Y
$$

where $\phi_{i}$ is defined by the deformation retraction.

(4) If $k_{i}=0,1,2$ then $\bar{e}^{k_{i}} / G_{i}$ is homeomorphic to a closed disc of dimension $k_{i}$. So attaching $\bar{e}^{k_{i}} / G_{i}$ is nothing but attaching a disc of dimension 0,1 or 2. Hence we may assume $G_{i}$ is trivial in these cases.

(5) Following Goresky Gor78 one may obtain a CW structure on an effective orbifold. Often however, it is too complicated for computational purpose.

Proposition 2.4. If $X$ is a $\mathbf{q}-C W$ complex, then $X$ is homotopic to a simplicial complex.

Proof. We prove it by induction. By definition of q-CW complex, $X_{0}$ has a simplicial complex structure. Suppose by induction, the space $X_{i-1}$ has a simplicial complex structure. Assume that $X_{i}$ is obtained by attaching a q-cell $\bar{e}^{k} / G_{i}$ via the map $\phi_{i}: S^{k-1} / G_{i} \rightarrow X_{i-1}$. Using Theorem 3.6 of Illman [Ill78], we get a simplicial complex structure on $\bar{e}^{k} / G_{i}$. Then by the simplicial approximation theorem ([Hat02, Theorem2C.1]), there is a homotopy from $\phi_{i}$ to a simplicial map $\xi_{i}: S^{k-1} / G_{i} \rightarrow X_{i-1}$. Then one can complete the proof by following the arguments in the proof of Theorem 2C.5 of [Hat02].

Now, we introduce the following definition of building sequence which enables us to compute the integral cohomology ring of certain spaces with q-CW complex structure and detect the existence of $p$-torsion.

Definition 2.5. Let $X$ be a finite $\mathbf{q}-\mathrm{CW}$ complex with $\ell$ many q-cells $e^{k_{i}} / G_{i}$ for $i \in\{1, \ldots, \ell\}$ and $1 \leq k_{i} \leq \operatorname{dim} X$. Let $Y_{i}$ be $i$-th stage q-CW subcomplex of $X$ and $0_{i}$ a free special point in $Y_{i}$. We say $\left\{\left(Y_{i}, 0_{i}\right)\right\}_{i=1}^{\ell}$ is a building sequence for $X$.

We recall that the finite group $G$ acts on $\bar{D}^{n}$ linearly. Thus the boundary $S^{n-1}$ is preserved by $G$-action. The quotient space $S^{n-1} / G$ is called an orbifold lens 
space in [BSS17]. We call a finite group $K$ a $|G|$-torsion if $K$ is trivial or the prime factors of the order $|K|$ is a subset of the prime factors of $|G|$. Now, the transfer homomorphism [Bor60, III.2] leads us the following lemma.

Proposition 2.6. The homology of an orbifold lens space $S^{n-1} / G$ is

$$
H_{j}\left(S^{n-1} / G ; \mathbb{Z}\right)= \begin{cases}\mathbb{Z} & \text { if } j=0, \\ a|G| \text {-torsion } & \text { if } 1 \leq j \leq n-2, \\ a|G| \text {-torsion or } \mathbb{Z} & \text { if } j=n-1 .\end{cases}
$$

In particular, if $G$-action preserves the orientation of $S^{n-1}$, then $H_{n-1}\left(S^{n-1} / G ; \mathbb{Z}\right) \cong$ $\mathbb{Z}$.

Proof. We see $H_{0}\left(S^{n-1} / G ; \mathbb{Z}\right) \cong \mathbb{Z}$ trivially. For $j=1, \ldots, n-2$, consider the following isomorphism

$$
H^{*}(X / G ; \mathbf{k}) \cong H^{*}(X ; \mathbf{k})^{G},
$$

where $X$ is a locally compact Hausdorff space, $G$ is a finite group and $\mathbf{k}$ is a field of characteristic zero or coprime to $|G|$, see [Bor60, III.2].

The result follows now from the universal coefficient theorem. In particular, if $G$-action preserves the orientation, then $H_{n-1}\left(S^{n-1} / G ; \mathbb{Z}\right) \cong \mathbb{Z}$ because $S^{n-1} / G$ is orientable.

The universal coefficient theorem leads to the following.

Corollary 2.7. If $p$ is coprime to the prime factors $p_{1}, \ldots, p_{r}$ of $|G|$, then the group $H_{*}\left(S^{n-1} / G ; \mathbb{Z}\right)$ has no $p$ torsion and $H_{j}\left(S^{n-1} / G ; \mathbb{Z}_{p}\right)$ is trivial if $j \neq 0, n-1$.

We end this section by discussing the degree of an attaching map. Let $X$ be a q-CW complex and $\phi: S^{k} / G \rightarrow X_{j} \subseteq X$ an attaching map where $X_{j}$ is the $j$-dimensional q-skeleton. Then $\phi$ induces homomorphisms

$$
\left(\phi_{*}\right)_{k}: H_{k}\left(S^{k} / G ; \mathbb{Z}\right) \rightarrow H_{k}\left(X_{j} ; \mathbb{Z}\right) .
$$

Now, $H_{k}\left(X_{j} ; \mathbb{Z}\right)$ is isomorphic to $\mathbb{Z}^{s} \oplus K$ for some non-negative integer $s$ and finite group $K$. By Proposition 2.6, we have $H_{k}\left(S^{k} / G ; \mathbb{Z}\right)$ is finite or $\mathbb{Z}$. The next definition of degree will play an important role in Section 5 .

Definition 2.8. Let $H_{k}\left(S^{k} / G ; \mathbb{Z}\right) \cong \mathbb{Z}$ and write $\left(\phi_{*}\right)_{k}(1)=\left(d_{1}, \ldots, d_{s}, x\right)$ for $1 \in \mathbb{Z}$. We define the degree of $\phi$ by

$$
\operatorname{deg} \phi:=\operatorname{gcd}\left\{d_{i} \mid 1 \leq i \leq s, d_{i} \neq 0\right\} .
$$

If all $d_{1}, \ldots, d_{s}$ are zero or $H_{k}\left(X_{j} ; \mathbb{Z}\right)$ has no free part, then we define degree of $\phi$ to be 0 .

\section{Integral homology of q-CW COMPLEXeS With CELLS IN EVEN DIMENSIONS}

This section is devoted to the proof of Theorem 1.1. which gives a sufficient condition for determining the torsion in the cohomology of certain families of finite q-CW complexes. Notice that one can verify Theorem 1.2 by applying Theorem 1.1 for each prime $p$. 
Proof of Theorem 1.1. We prove the claim by the induction on the free special points in the sequence $\left\{\left(Y_{i}, 0_{i}\right)\right\}_{i=1}^{\ell}$. First, notice that if $i=1, Y_{i}$ is a point and if $i=2$ then $Y_{i}$ is a $\mathbf{q}-\mathrm{CW}$ complex obtained by attaching an even dimensional q-cell to a point. That is, $Y_{2}$ is homeomorphic to a suspension on $S^{2 k_{i}-1} / G_{i}$. Then by Proposition 2.6, the claim is true.

Now we assume that $H_{*}\left(Y_{i-1} ; \mathbb{Z}\right)$ has no $p$-torsion and $H_{\text {odd }}\left(Y_{i-1} ; \mathbb{Z}_{p}\right)$ is trivial for $i>1$. To complete the induction, we shall prove that the same holds for $Y_{i}$. Note that $Y_{i}$ can be constructed from $Y_{i-1}$ by attaching a q-cell $\bar{e}^{2 k_{i}} / G_{i}$ to it. So we have the cofibration:

$$
S^{2 k_{i}-1} / G_{i} \longrightarrow Y_{i-1} \longrightarrow Y_{i} .
$$

This cofibration induces the following long exact sequence in homology,

$$
\begin{gathered}
\cdots \longrightarrow H_{j+1}\left(Y_{i}\right) \longrightarrow \widetilde{H}_{j}\left(S^{2 k_{i}-1} / G_{i}\right) \longrightarrow \\
H_{j}\left(Y_{i-1}\right) \longrightarrow H_{j}\left(Y_{i}\right) \longrightarrow \widetilde{H}_{j-1}\left(S^{2 k_{i}-1} / G_{i}\right) \longrightarrow \cdots .
\end{gathered}
$$

Suppose that $j$ is odd. By the induction hypothesis, the group $H_{j}\left(Y_{i-1} ; \mathbb{Z}\right)$ has no $p$-torsion and $H_{j}\left(Y_{i-1} ; \mathbb{Z}_{p}\right)$ is trivial. On the other hand, $\widetilde{H}_{j-1}\left(S^{2 k_{i}-1} / G_{i} ; \mathbb{Z}\right)$ has no $p$-torsion and $H_{j-1}\left(S^{2 k_{i}-1} / G_{i} ; \mathbb{Z}_{p}\right)$ is trivial by Corollary 2.7. Therefore, from $(3.2)$ we have $H_{j}\left(Y_{i} ; \mathbb{Z}\right)$ has no $p$-torsion and $H_{j}\left(Y_{i} ; \mathbb{Z}_{p}\right)$ is trivial.

Next, we assume that $j$ is even. Then, in the exact sequence (3.2), the groups $\widetilde{H}_{j-1}\left(S^{2 k_{i}-1} / G_{i} ; \mathbb{Z}\right), H_{j}\left(Y_{i-1} ; \mathbb{Z}\right)$ and $\widetilde{H}_{j}\left(S^{2 k_{i}-1} / G_{i} ; \mathbb{Z}\right)$ have no $p$-torsion. Therefore $H_{j}\left(Y_{i} ; \mathbb{Z}\right)$ has no $p$-torsion. This completes the induction.

\section{Applications and Examples}

The goal of this section is to illustrate the results of Section 3 with some broad applications which improve upon certain previous results.

4.1. Toric orbifolds. Toric orbifolds were introduced in DJ91 and explicitly studied in PS10 $^{2}$. In [BSS17, the authors introduced the concept of a retraction sequence $3^{3}$ for a simple polytope and discussed the integral homology and cohomology of toric orbifolds. In this subsection we show that a retraction sequence determines a q-CW structure on the toric orbifold and then we compare the main theorem of BSS17, Theorem 1.1] and Theorem 1.2. For convenience we adhere the notation of [BSS17.

We begin by summarizing the definition of a retraction sequence of a simple polytope. Given an $n$-dimensional simple polytope $Q$ with $\ell$ vertices, we construct a sequence of triples $\left\{\left(B_{k}, E_{k}, b_{k}\right)\right\}_{1 \leq k \leq \ell}$, inductively.

The first term is defined by $B_{1}=Q=E_{1}$ and $b_{1}$ is a vertex of $B_{1}$. Next, given $(k-1)$-th term $\left(B_{k-1}, E_{k-1}, b_{k-1}\right)$, the next term $\left(B_{k}, E_{k}, b_{k}\right)$ is defined by setting

$$
B_{k}=\bigcup\left\{E \mid E \text { is a face of } B_{k-1} \text { and } b_{k-1} \notin V(E)\right\} \text {. }
$$

\footnotetext{
${ }^{2}$ After DJ91, toric manifold was renamed in BP02 as quasitoric manifold, and the authors of [PS10] used quasitoric orbifolds instead of toric orbifolds of [DJ91].

${ }^{3}$ Retraction sequences have strong connection with shellability of a simplicial complex. The authors of [BSS17] are preparing a separated paper about this.
} 
If a vertex $b_{k}$ exists in $B_{k}$ satisfying that $b_{k}$ has a neighborhood homeomorphic to $\mathbb{R}_{\geq 0}^{d}$ as manifold with corners for some $d \in\left\{1, \ldots, \operatorname{dim} B_{k}\right\}$, then we let $E_{k}$ be the unique face containing $b_{k}$ with $\operatorname{dim} E_{k}=d$. We call $b_{k}$ a free vertex of $B_{k}$. Such a sequence is called a retraction sequence for $Q$ if the sequence ends up with $\left(B_{\ell}, E_{\ell}, b_{\ell}\right)$ such that $B_{\ell}=E_{\ell}=b_{\ell}$ for some vertex $b_{\ell} \in V(Q)$.

We call a simple polytope $Q$ admissible if there exists at least one free vertex in each polytopal subcomplex $B_{k}$ defined in (4.1). Hence, given $k$-th term $B_{k}$ as defined in (4.1), any choice of free vertex of $B_{k}$ defines a retraction sequence. Two different retraction sequences of a prism are described in Figure 1 below, which shows that the prism is admissible by its symmetry.

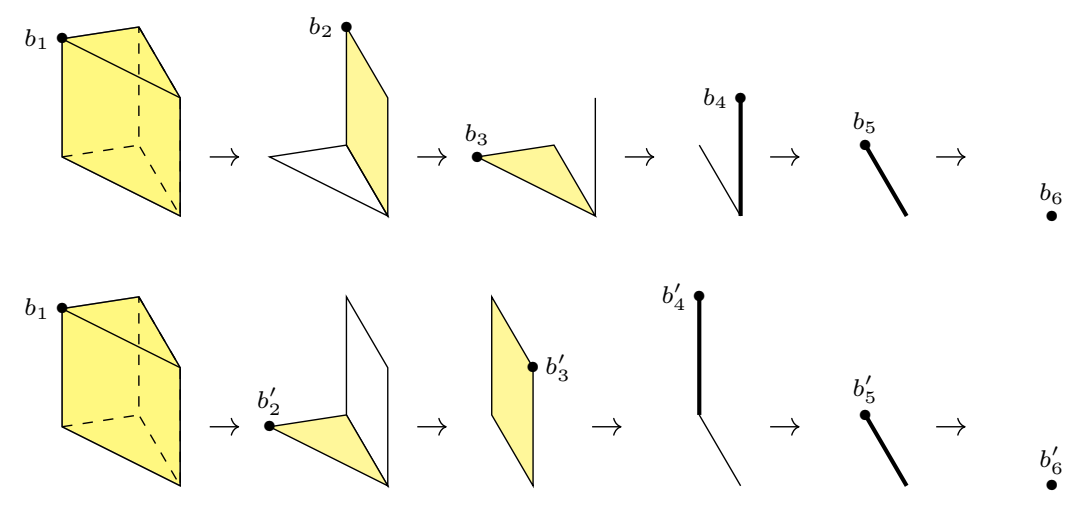

FiguRE 1. Two retraction sequences.

Next, we recall briefly the construction of toric orbifolds. A toric orbifold is constructed from a combinatorial pair $(Q, \lambda)$, called an $\mathcal{R}$-characteristic pair, of an $n$-dimensional simple convex polytope $Q$ and a function

$$
\lambda: \mathcal{F}(Q)=\left\{F_{1}, \ldots, F_{m}\right\} \rightarrow \mathbb{Z}^{n},
$$

where $\mathcal{F}(Q)$ is the set of codimension one faces of $Q$, called facets, and $\lambda$ satisfies the following condition:

$$
\left\{\lambda\left(F_{i_{1}}\right), \ldots, \lambda\left(F_{i_{k}}\right)\right\} \text { is linearly independent, whenever } \bigcap_{j=1}^{k} F_{i_{j}} \neq \emptyset .
$$

We call such a function $\lambda$ an $\mathcal{R}$-characteristic function on $Q$. For the notational convenience, we sometimes denote $\lambda_{i}=\lambda\left(F_{i}\right)$ and call it an $\mathcal{R}$-characteristic vector assigned to the facet $F_{i}$.

Remark 4.1. One example of such a function can be provided by a simple lattice polytope which is a simple polytope obtained by the convex hull of finitely many lattice points in $\mathbb{Z}^{n} \subset \mathbb{R}^{n}$. One can naturally assign each facet of simple lattice polytope its primitive normal vector as an $\mathcal{R}$-characteristic vector. Such a polytope is one of the key combinatorial objects in toric geometry, as we shall explain briefly in the next subsection.

We may regard $\mathbb{Z}^{n}$, the target space of $\lambda$, as the $\mathbb{Z}$-submodule of the Lie algebra of $T^{n}$. Let $E(x)=F_{i_{1}} \cap \cdots \cap F_{i_{k}}$ denote the face with $x$ in its (relative) interior, 
and $T_{E(x)} \in T^{n}$ the subtorus generated by the images of $\lambda\left(F_{i_{1}}\right), \ldots, \lambda\left(F_{i_{k}}\right)$ under the $\operatorname{map} \operatorname{span}_{\mathbb{Z}}\left\{\lambda_{i_{1}}, \ldots, \lambda_{i_{k}}\right\} \hookrightarrow \mathbb{Z}^{n} \stackrel{\exp }{\longrightarrow} T^{n}$. Then, given an $\mathcal{R}$-characteristic pair $(Q, \lambda)$, we construct the following quotient space

$$
X(Q, \lambda):=Q \times T^{n} / \sim_{\lambda},
$$

where $(x, t) \sim_{\lambda}(y, s)$ if and only if $x=y$ and $t^{-1} s \in T_{E(x)}$.

Now $X(Q, \lambda)$ has an orbifold structure induced by the group operation as described in [PS10, Section 2]. The natural $T^{n}$-action, given by the multiplication on the second factor, induces the orbit map

$$
\pi: X(Q, \lambda) \rightarrow Q
$$

defined by the projection onto the first factor. The space $X(Q, \lambda)$ is called the toric orbifold associated to an $\mathcal{R}$-characteristic pair $(Q, \lambda)$. We remark that the authors of PS10] gave an axiomatic definition of toric orbifolds, which generalizes the definition of toric manifolds of [DJ91, Section 1].

Notice that the preimage $\pi^{-1}\left(F_{i}\right)$ of a facet $F_{i}$ is a codimension 2 subspace fixed by a circle subgroup of $T^{n}$ generated by $\lambda_{i}$. Moreover, the preimage $\pi^{-1}(E)$ of a face $E=F_{i_{1}} \cap \cdots \cap F_{i_{k}}$ is the intersection of $\pi^{-1}\left(F_{i_{1}}\right), \ldots, \pi^{-1}\left(F_{i_{k}}\right)$, which is again $T^{n}$-invariant. Indeed, it is shown in [PS10, Section 2.3] that $\pi^{-1}(E)$ is also a toric orbifold for each face $E$ of $Q$, whose $\mathcal{R}$-characteristic pair is described below.

As above, let $E=F_{i_{1}} \cap \cdots \cap F_{i_{k}}$ be a face of codimension $k$ in $Q$, where $F_{i_{1}}, \ldots, F_{i_{k}}$ are facets. One can define a natural projection

$$
\rho_{E}: \mathbb{Z}^{n} \rightarrow \mathbb{Z}^{n} /\left(\left(\operatorname{span}_{\mathbb{Z}}\left\{\lambda_{i_{1}}, \ldots, \lambda_{i_{k}}\right\} \otimes_{\mathbb{Z}} \mathbb{R}\right) \cap \mathbb{Z}^{n}\right),
$$

where $\left(\operatorname{span}_{\mathbb{Z}}\left\{\lambda_{i_{1}}, \ldots, \lambda_{i_{k}}\right\} \otimes_{\mathbb{Z}} \mathbb{R}\right) \cap \mathbb{Z}^{n}$ is a free $\mathbb{Z}$-module of rank $k$. We regard $E$ as an independent simple polytope whose facets $\mathcal{F}(E)$ are of the form:

$$
\mathcal{F}(E)=\left\{E \cap F_{j} \mid F_{j} \in \mathcal{F}(Q) \text { and } j \notin\left\{i_{1}, \ldots, i_{k}\right\} \text {, and } E \cap F_{j} \neq \varnothing\right\} .
$$

Now, the composition of the maps $\rho_{E}$ and $\lambda$ yields an $\mathcal{R}$-characteristic function for $E$

$$
\lambda_{E}: \mathcal{F}(E) \rightarrow \mathbb{Z}^{n-k},
$$

defined for $\lambda_{E}\left(E \cap F_{j}\right)$ to be the primitive vector of $\left(\rho_{E} \circ \lambda\right)\left(F_{j}\right)$. Indeed, condition 4.2 for the $\mathcal{R}$-characteristic function $\lambda_{E}$ follows naturally from $\lambda$.

Next, we define certain integers associated to each vertex. Let $v$ be a vertex of $Q$ and $E$ a face of codimension $k$ containing $v$. Then, there are facets $F_{j_{1}}, \ldots, F_{j_{n-k}}$ such that $v=\left(E \cap F_{j_{1}}\right) \cap \cdots \cap\left(E \cap F_{j_{n-k}}\right)$. We define

$$
g_{E}(v):=\left|\operatorname{det}\left[\lambda_{E}\left(E \cap F_{j_{1}}\right)^{t}, \ldots, \lambda_{E}\left(E \cap F_{j_{n-k}}\right)^{t}\right]\right| \text {, }
$$

where $t$ denotes the transpose. In particular, when $E=Q$ and $v=F_{i_{1}} \cap \cdots \cap F_{i_{n}}$, the number $g_{Q}(v)$ is

$$
g_{Q}(v)=\left|\operatorname{det}\left[\lambda\left(F_{i_{1}}\right)^{t} \cdots \lambda\left(F_{i_{n}}\right)^{t}\right]\right| .
$$

Given an $\mathcal{R}$-characteristic pair $(Q, \lambda)$ and each term $\left(B_{i}, E_{i}, b_{i}\right)$ of a retraction sequence of $Q$, we denote naturally $g_{B_{i}}(v):=g_{E_{i}}(v)$ for each free vertex $v$ of $B_{i}$, which makes the statement of Theorem 4.3 simpler.

Remark 4.2. The number $g_{E}(v)$ encodes the singularity of $X\left(E, \lambda_{E}\right)$. Indeed, the local group of the orbifold chart around the point $\pi_{E}^{-1}(v)$, where $\pi_{E}: X\left(E, \lambda_{E}\right) \rightarrow E$ is the orbit map, has the order $g_{E}(v)$. 
The next theorem gives a sufficient condition ensuring that the cohomology of a toric orbifold is concentrated in even degree and torsion free.

Theorem 4.3. BSS17, Theorem 1.1] Given any toric orbifold $X(Q, \lambda)$ over an admissible simple polytope, assume that

$$
\operatorname{gcd}\left\{g_{B_{i}}(v) \mid v \text { is a free vertex in } B_{i}\right\}=1 \text {, }
$$

for each $B_{i}$ which appears in a retraction sequence of $Q$ with $\operatorname{dim} B_{i}>1$; then $H^{*}(X(Q, \lambda) ; \mathbb{Z})$ is torsion free and concentrated in even degrees.

We remark that BSS17, Theorem 1.1] was proved for toric orbifolds over admissible simple polytopes, but it was not mentioned explicitly. Retraction sequences and building sequences are related by the following.

Proposition 4.4. If $\left\{\left(B_{i}, E_{i}, b_{i}\right)\right\}_{i=1}^{\ell}$ is a retraction sequence of an $n$-dimensional simple polytope $Q$ and $(Q, \lambda)$ is an $\mathcal{R}$-characteristic pair, then it induces a building sequence $\left\{\left(Y_{i}, 0_{i}\right)\right\}_{i=1}^{\ell}$ of $X(Q, \lambda)$.

Proof. Let $E_{i}$ be a face of $Q$ with $\operatorname{dim} E_{i}=n-k$, and $U_{i}$ the open subset of $E_{i}$ obtained by deleting the faces of $E_{i}$ not containing the vertex $b_{i}$. Observe that $b_{i}=$ $\bigcap_{j=1}^{n-k}\left(E_{i} \cap F_{i_{j}}\right)$ for some facets $F_{i_{1}}, \ldots, F_{i_{n-k}}$ of $Q$ and $U_{i}$ is homeomorphic to $\mathbb{R}_{\geq 0}^{n-k}$ as manifold with corners. Note that $\pi^{-1}\left(E_{i}\right)$ is a toric orbifold and homeomorphic to $X\left(E_{i}, \lambda_{E_{i}}\right)$, see BSS17, Proposition 3.3]. So, we can apply the orbifold chart construction on a toric orbifold described in [PS10, Subsection 2.1] to get that $\pi^{-1}\left(U_{i}\right)$ is homeomorphic to the quotient of an open $\operatorname{disc} D^{2(n-k)}$ by a finite group $G_{i}$ which is given by

$$
G_{i}=\mathbb{Z}^{n-k} / \operatorname{span}_{\mathbb{Z}}\left\{\lambda_{E_{i}}\left(E_{i} \cap F_{i_{1}}\right), \ldots, \lambda_{E_{i}}\left(E_{i} \cap F_{i_{n-k}}\right)\right\} .
$$

Since $Q=\bigcup_{i=1}^{\ell} U_{i}$, we get a q-CW complex structure on $X(Q, \lambda)$ with q-cells $\pi^{-1}\left(U_{i}\right)$ for $i=1, \ldots, \ell$. Setting $Y_{i}=\bigcup_{j \geq \ell-i+1}\left(\pi^{-1}\left(U_{j}\right)\right)$ gives a building sequence $\left\{\left(Y_{i}, 0_{i}\right)\right\}_{i=1}^{\ell}$ where $0_{i}=\pi^{-1}\left(b_{i}\right)$ for $i=1, \ldots, \ell$.

We note that the order $\left|G_{i}\right|$ is exactly $g_{E_{i}}\left(b_{i}\right)$ defined as in 4.7).

Proposition 4.5. If a toric orbifold $X(Q, \lambda)$ satisfies the assumption of Theorem 4.3, then it satisfies the assumption of Theorem 1.2.

Proof. Let $X(Q, \lambda)$ be a toric orbifold over an $n$-dimensional admissible simple polytope $Q$ with $\ell$ vertices, and $p$ a prime. Since $B_{1}=Q$, we have

$$
\operatorname{gcd}\left\{g_{Q}(v) \mid v \in V(Q)\right\}=1
$$

and so there is a $b_{1} \in V(Q) \operatorname{such}$ that $\operatorname{gcd}\left\{p, g_{Q}\left(b_{1}\right)\right\}=1$. We consider $\left(B_{1}, E_{1}, b_{1}\right)=$ $\left(Q, Q, b_{1}\right)$ for the first step of the retraction sequence. Since $Q$ is simple, $B_{2}$ has $n$ many free vertices, say $\left\{v_{i_{1}}, \ldots, v_{i_{n}}\right\}$, namely, the ones at the ends of the $n$ deleted edges joining $b_{1}$. The assumption of Theorem 4.3 implies that

$$
\operatorname{gcd}\left\{g_{B_{2}}\left(v_{i_{1}}\right), \ldots, g_{B_{2}}\left(v_{i_{n}}\right)\right\}=1,
$$

which guarantees that there is a vertex $b_{2} \in\left\{v_{i_{1}}, \ldots, v_{i_{n}}\right\}$ with $\operatorname{gcd}\left\{p, g_{B_{2}}\left(b_{2}\right)\right\}=1$. Let $E_{2}$ be the face of $B_{1}=Q$ determined by the edges of $B_{2}$ which intersect $b_{2}$. So $\left(B_{2}, E_{2}, b_{2}\right)$ can be a second term of a retraction sequence. Since $Q$ is an admissible simple polytope, then $B_{3}$ has at least one free vertex. Continuing this process, since the number of vertices $\ell=|V(Q)|$ is finite, one get a retraction $\left\{\left(B_{i}, E_{i}, b_{i}\right)\right\}_{i=1}^{\ell}$ of 
$Q$ such that $\operatorname{gcd}\left\{p, g_{E_{i}}\left(b_{i}\right)\right\}=1$ for $i=1, \ldots, \ell$. Therefore the result follows from Proposition 4.4 and its proof.

The next theorem is the direct application of Theorem 1.2 to toric orbifolds.

Theorem 4.6. Let $X(Q, \lambda)$ be a toric orbifold. If, for each prime $p$, there is a retraction sequence $\left\{\left(B_{i}, E_{i}, v_{i}\right)\right\}_{i=1}^{\ell}$ of $Q$ such that $\operatorname{gcd}\left\{p, g_{E_{i}}\left(v_{i}\right)\right\}=1$, then $H^{*}(X(Q, \lambda) ; \mathbb{Z})$ is torsion free and concentrated in even degrees.

Proof. The proof follows from Proposition 4.4 and Theorem 1.2

The next example illustrates the fact that the converse of Proposition 4.5 is not true in general. Hence, Theorem 4.6 generalizes Theorem 4.3 .

Example 4.7. In this example we construct a toric orbifold which satisfies the condition of Theorem 4.6, but fails to satisfy the hypothesis of Theorem 4.3 . Consider the $\mathcal{R}$-characteristic pair $(Q, \lambda)$ illustrated in Figure 2.

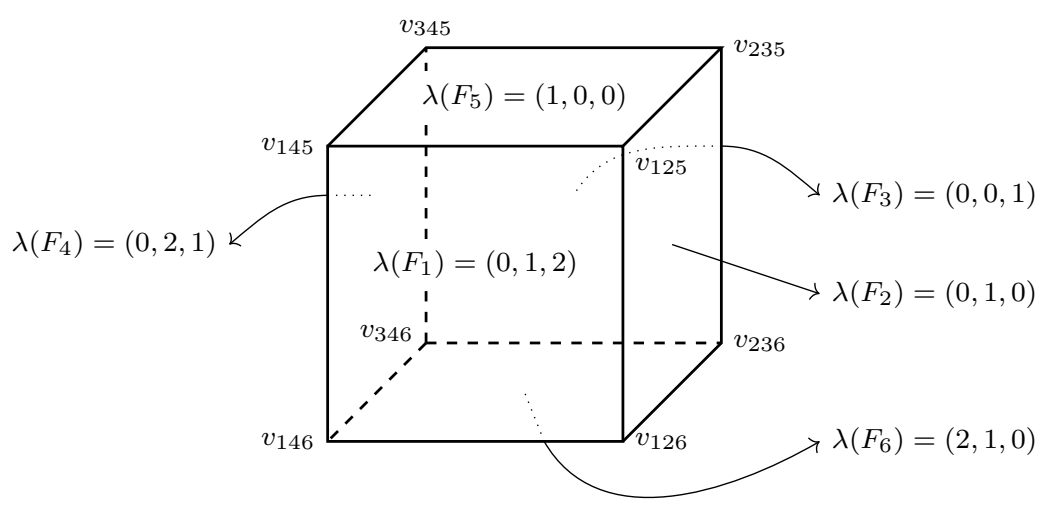

Figure 2. An $\mathcal{R}$-characteristic function on 3-cube $Q$.

One can compute that

$$
\begin{aligned}
& g_{Q}\left(v_{125}\right)=2, g_{Q}\left(v_{235}\right)=1, g_{Q}\left(v_{345}\right)=2, g_{Q}\left(v_{145}\right)=3, \\
& g_{Q}\left(v_{126}\right)=4, g_{Q}\left(v_{236}\right)=2, g_{Q}\left(v_{346}\right)=4, g_{Q}\left(v_{146}\right)=6 .
\end{aligned}
$$

Now we consider two different retraction sequences of $Q$, as in Figure 3 . The first retraction sequence is given by

$$
\begin{aligned}
& \left(B_{1}, B_{1}, v_{235}\right) \rightarrow\left(B_{2}, F_{6}, v_{236}\right) \rightarrow\left(B_{3}, F_{4}, v_{345}\right) \rightarrow\left(B_{4}, F_{1}, v_{125}\right) \rightarrow \\
& \left(B_{5}, F_{4} \cap F_{6}, v_{346}\right) \rightarrow\left(B_{6}, F_{1} \cap F_{4}, v_{145}\right) \rightarrow\left(B_{7}, F_{1} \cap F_{6}, v_{126}\right) \rightarrow \\
& \left(B_{8}, v_{146}, v_{146}\right),
\end{aligned}
$$

which is illustrated in Figure 3 Retraction (a). In the second term of the sequence 4.9), we compute $g_{F_{6}}\left(v_{236}\right)$ as follows; since

$$
\left(\operatorname{span}\left\{\lambda\left(F_{6}\right)\right\} \otimes_{\mathbb{Z}} \mathbb{R}\right) \cap \mathbb{Z}^{3}=\operatorname{span}\{(2,1,0)\},
$$

a choice of basis $\{(1,0,0),(2,1,0),(0,0,1)\}$ of $\mathbb{Z}^{3}$ induces the $\mathcal{R}$-characteristic function

$$
\lambda_{F_{6}}: \mathcal{F}\left(F_{6}\right)=\left\{F_{i} \cap F_{6} \mid i=1,2,3,4\right\} \rightarrow \mathbb{Z}^{3} / \operatorname{span}\{(2,1,0)\} \cong \mathbb{Z}^{2},
$$




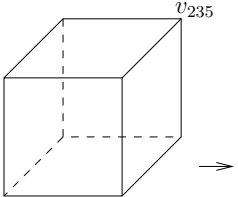

$B_{1}$

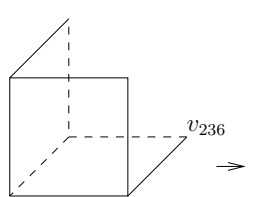

$B_{2}$

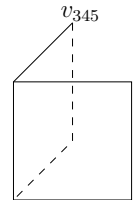

$B_{3}$

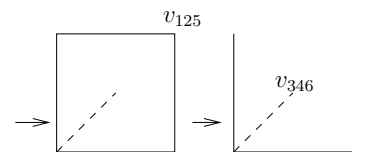

$B_{4}$

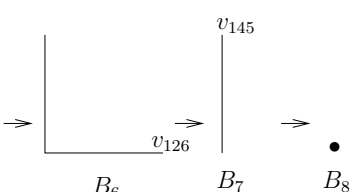

Retraction sequence (a)

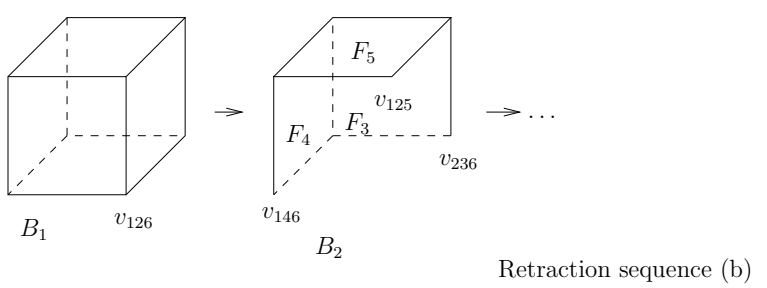

Figure 3. Two retraction sequences of $Q$.

on $F_{6}$ defined as in 4.6$)$ by $\lambda_{F_{6}}\left(F_{1} \cap F_{6}\right)=(-1,1), \lambda_{F_{6}}\left(F_{2} \cap F_{6}\right)=(-1,0)$, $\lambda_{F_{6}}\left(F_{3} \cap F_{6}\right)=(0,1)$ and $\lambda_{F_{6}}\left(F_{4} \cap F_{6}\right)=(-4,1)$. In particular,

$$
g_{F_{6}}\left(v_{236}\right)=\left|\operatorname{det}\left[\lambda_{F_{6}}\left(F_{2} \cap F_{6}\right)^{t} \quad \lambda_{F_{6}}\left(F_{3} \cap F_{6}\right)^{t}\right]\right|=\left|\operatorname{det}\left[\begin{array}{cc}
-1 & 0 \\
0 & 1
\end{array}\right]\right|=1 .
$$

Similarly, one can compute the integers defined in 4.7 with respect to the retraction sequence (4.9) as follows:

$$
g_{F_{4}}\left(v_{345}\right)=g_{F_{1}}\left(v_{125}\right)=g_{F_{4} \cap F_{6}}\left(v_{346}\right)=g_{F_{1} \cap F_{4}}\left(v_{145}\right)=g_{F_{1} \cap F_{6}}\left(v_{126}\right)=1 .
$$

Hence, the $\mathcal{R}$-characteristic pair $(Q, \lambda)$ defined in Figure 2 satisfies the hypothesis of Theorem 4.6 .

Next, we consider a retraction sequence whose first choice of free vertex is $v_{126}$, see Figure 3 -Retraction (b). The vertices $v_{125}, v_{146}, v_{236}$ are free vertices in $B_{2}$. Similarly as above, one can compute that $g_{F_{4}}\left(v_{146}\right)=2, g_{F_{5}}\left(v_{125}\right)=2$, and $g_{F_{3}}\left(v_{236}\right)=2$. Hence,

$$
\operatorname{gcd}\left\{g_{F_{4}}\left(v_{146}\right), g_{F_{5}}\left(v_{125}\right), g_{F_{3}}\left(v_{236}\right)\right\}=2,
$$

which fails to satisfy the assumption of Theorem 4.3 for $B_{2}$.

4.2. Toric varieties. Toric varieties are important objects in algebraic geometry which have been studied from the beginning of nineteen-seventies. They featured prominently in early investigations of the phenomenon of mirror symmetry. One approach to constructing them begins with a lattice $N\left(\cong \mathbb{Z}^{n}\right)$ and a fan $\Sigma$ which is a finite collection of cones in a vector space $N_{\mathbb{R}}:=N \otimes_{\mathbb{Z}} \mathbb{R}\left(\cong \mathbb{R}^{n}\right)$ satisfying certain conditions, see [CLS11, Chapter 1-3], [Ewa96, Chapter 5], Ful93, Chapter 1] and Oda88, Chapter 1] for details.

We begin by reviewing briefly the definition of cones and fans. A cone is a positive hull of finitely many elements in $N_{\mathbb{R}}$. To be more precise, for a finite subset $S=\left\{v_{1}, \ldots, v_{k}\right\} \subset N_{\mathbb{R}}$, the cone of $S$ is a subset of $N_{\mathbb{R}}$ of the form

$$
\sigma:=\operatorname{Cone}(S)=\left\{\sum_{i=1}^{k} c_{i} v_{i} \mid c_{i} \geq 0\right\} \text {. }
$$


To each cone $\sigma=$ Cone $(S)$, one can associate a dual cone $\sigma^{\vee} \subset M_{\mathbb{R}}$, where $M_{\mathbb{R}}$ is a vector space dual to $N_{\mathbb{R}}$, defined as follows:

$$
\sigma^{\vee}=\left\{y \in M_{\mathbb{R}} \mid\langle y, v\rangle \geq 0 \text { for all } v \in \sigma\right\} .
$$

One can easily see that $\left(\sigma^{\vee}\right)^{\vee}=\sigma$.

A cone $\sigma_{1}:=\operatorname{Cone}\left(S_{1}\right)$ is called a face of another cone $\sigma_{2}:=\operatorname{Cone}\left(S_{2}\right)$ if there is a vector $w \in \sigma_{2}^{\vee}$ and corresponding hyperplane $H_{w}:=\left\{x \in N_{\mathbb{R}} \mid\langle w, x\rangle=0\right\}$ such that $\sigma_{1}=H_{w} \cap \sigma_{2}$ and $\sigma_{2}$ lie in the positive half space $H_{w}^{+}:=\left\{x \in N_{\mathbb{R}} \mid\langle w, x\rangle \geq 0\right\}$. Notice that $\sigma_{2}^{\vee}$ is a face of $\sigma_{1}^{\vee}$, if $\sigma_{1}$ is a face of $\sigma_{2}$.

The set of one dimensional face of a cone $\sigma$ is called the generating rays of $\sigma$. A cone $\sigma$ is called strongly convex if it does not contain a nontrivial linear subspace of $N_{\mathbb{R}}$, and is called simplicial if its generating rays are linearly independent in $N_{\mathbb{R}}$.

Let $M$ be the lattice dual to $N$. The lattice points $S_{\sigma}:=\sigma^{\vee} \cap M$ form a finitely generated semigroup (see CLS11, Proposition 1.2.17]) which yields an algebraic variety

$$
U_{\sigma}:=\operatorname{Spec}\left(\mathbb{C}\left[S_{\sigma}\right]\right)
$$

which is called the affine toric variety associated to a cone $\sigma$.

Remark 4.8. Every affine toric variety $U_{\sigma}$ associated to a cone $\sigma \in N_{\mathbb{R}}$ equips with the action of $\left(\mathbb{C}^{*}\right)^{n}$, which is obtained by identifying $\left(\mathbb{C}^{*}\right)^{n} \cong \operatorname{Spec}(\mathbb{C}[M])$. Here we regard $M$ as the dual cone of $\{0\} \in N_{\mathbb{R}}$. See [CLS11, Chapter 1] or [Ful93, Section 1.3].

A fan $\Sigma$ in $N_{\mathbb{R}}$ is a finite collection of strongly convex cones such that

(1) if $\sigma_{2} \in \Sigma$ and $\sigma_{1}$ is a face of $\sigma_{2}$, then $\sigma_{1} \in \Sigma$;

(2) if $\sigma_{1}, \sigma_{2} \in \Sigma$, then $\sigma_{1} \cap \sigma_{2}$ is a face of both $\sigma_{1}$ and $\sigma_{2}$.

One natural way of defining a fan in toric geometry is to use a full dimensional lattice polytope $Q \subset M_{\mathbb{R}}$, see Remark 4.1. To be more precise, let $\mathcal{F}(Q)=\left\{F_{1}, \ldots, F_{m}\right\}$ be facets of $Q$ as in Subsection 4.1. For each $i$, let $\lambda_{i} \in N$ denote the vector such that

$$
Q=\left\{y \in M_{\mathbb{R}} \mid\left\langle y, \lambda_{i}\right\rangle+a_{i} \geq 0 \text { for some } a_{i} \in \mathbb{Z} \text { with } i=1, \ldots, m\right\} .
$$

Observe that $\lambda_{i}$ in 4.10 represents the inward normal vector of the facet

$$
F_{i}=\left\{y \in M_{\mathbb{R}} \mid\left\langle y, \lambda_{i}\right\rangle+a_{i}=0\right\} \cap Q
$$

for $i=1, \ldots, m$. Let a face $E$ of $Q$ be given by $E=F_{i_{1}} \cap \cdots \cap F_{i_{k}}$ for some facets $F_{i_{1}}, \ldots, F_{i_{k}}$. Then we can associate a cone $\sigma_{E}$ to each face $E$ of $Q$ as follows:

$$
\sigma_{E}=\text { Cone }\left\{\lambda_{i_{1}}, \ldots, \lambda_{i_{k}}\right\} \text {. }
$$

The fan $\Sigma_{Q}$ associated to a lattice polytope $Q$, which is called the normal fan of $Q$, is the collection $\left\{\sigma_{E} \mid E\right.$ is a face of $\left.Q\right\}$ whose face structure naturally follows from the face structure of $Q$. In general, we call a fan $\Sigma$ polytopal if $\Sigma$ is a normal fan of a lattice polytope.

Finally, the toric variety $X_{\Sigma}$ corresponding to a fan $\Sigma$ is defined by taking disjoint union $\bigsqcup_{\sigma \in \Sigma} U_{\sigma}$ and the gluing is by rational maps determined by the generating rays of two cones $\sigma_{1}$ and $\sigma_{2}$ with nonempty intersections. In particular, the torus $\left(\mathbb{C}^{*}\right)^{n} \cong U_{\{0\}} \subset U_{\sigma}$ of each affine toric variety is identified by the gluing, so yields the action of $\left(\mathbb{C}^{*}\right)^{n}$ on $X_{\Sigma}$. Further details may be found in [Ful93, Section 1.4]. 
It is well-known from literature, such as CLS11, Ewa96, Ful93, that a fan $\Sigma$ is polytopal if and only if the corresponding toric variety $X_{\Sigma}$ is projective, and $\Sigma$ is simplicial, i.e., $\Sigma$ consists of simplicial cones, if and only if $X_{\Sigma}$ is an orbifold.

Observe that if a polytopal fan $\Sigma$ is simplicial, then the corresponding polytope $Q$ is simple. Moreover, the primitive inward normal vector $\lambda_{i}$ of each facet $F_{i} \in \mathcal{F}(Q)$ satisfies the condition 4.2 since $Q$ is a simple lattice polytope. Hence, such a fan $\Sigma$ naturally yields an $\mathcal{R}$-characteristic pair $(Q, \lambda)$, introduced in Subsection 4.1 , by defining $\lambda\left(F_{i}\right)$ to be the primitive inward normal vector of a facet $F_{i} \in \mathcal{F}(Q)$, see Remark 4.1.

In particular, if an $\mathcal{R}$-characteristic pair $(Q, \lambda)$ is defined from a polytopal fan $\Sigma$ as above, then one can show that there is a $T^{n}$-equivariant homeomorphism

$$
X(Q, \lambda) \cong X_{\Sigma},
$$

where $T^{n}$-action on $X(Q, \lambda)$ is the multiplication on the second factor (see (4.3p) and $T^{n}$-action on $X_{\Sigma}$ is provided by regarding $T^{n}$ as a compact torus in $\left(\mathbb{C}^{*}\right)^{n}$, see [CLS11, Chapter 12] and [DJ91, Section 7]. Hence, one can apply theorems of Subsection 4.1 to a projective toric variety from a simplicial fan, i.e., a toric variety from a normal fan of a simple lattice polytope.

Recently, the authors of BSS17] computed the integral cohomology ring of certain toric varieties associated to a normal fan $\Sigma$ of a simple polytope $Q$. The description was given in terms of the weighted Stanley-Reisner ring $w \mathcal{S R}[\Sigma]$ of a simplicial fan $\Sigma$, which is a certain subring of the usual Stanley-Reisner ring $\mathcal{S R}[Q]=\mathbb{Z}\left[x_{1}, \ldots, x_{m}\right] / \mathcal{I}$, see [BSS17, (5.3)]. This subring is determined by an integrality condition which encodes the singularity of each fixed point. Then, assuming $H^{\text {odd }}\left(X_{\Sigma} ; \mathbb{Z}\right)=0$, it was shown that there is an isomorphism between $H^{*}\left(X_{\Sigma} ; \mathbb{Z}\right)$ and $w \mathcal{S} \mathcal{R}[\Sigma] / \mathcal{J}$, where $\mathcal{J}$ is an ideal generated by the linear relations in 4.12 , which is determined by the geometry of $\Sigma$. Hence, by the aid of Theorem 1.1. we get the following theorem which generalizes a result of [BSS17, Theorem $5.3]$.

Theorem 4.9. Let $X_{\Sigma}$ be a projective toric variety associated to a normal fan $\Sigma$ of a simple polytope $Q$ with $m$ facets. If for each prime $p$ there is a retraction sequence $\left\{\left(B_{i}, E_{i}, v_{i}\right)\right\}_{i=1}^{\ell}$ of $Q$ such that $\operatorname{gcd}\left\{p, g_{E_{i}}\left(v_{i}\right)\right\}=1$, then the cohomology ring of $X_{\Sigma}$ is

$$
H^{*}\left(X_{\Sigma} ; \mathbb{Z}\right) \cong w \mathcal{S R}[\Sigma] / \mathcal{J} \subseteq \mathbb{Z}\left[x_{1}, \ldots, x_{m}\right] / \mathcal{I}+\mathcal{J},
$$

where $\mathcal{I}$ is the Stanley-Reisner ideal of $Q$ and $\mathcal{J}$ is the ideal generated by linear relations

$$
\sum_{i=1}^{m}\left\langle\lambda_{i}, \mathbf{e}_{j}\right\rangle x_{i}=0, \quad j=1, \ldots, n
$$

where $\operatorname{deg} x_{i}=2$, $\mathbf{e}_{j}$ denotes the $j$-th standard unit vector in $\mathbb{Z}^{n}$ and $\lambda_{i}$ is defined by 4.10 .

4.3. Torus orbifolds. A torus orbifold is a $2 n$-dimensional closed orbifold with an action of the $n$-dimensional real torus with non-empty fixed points. Introduced by Hattori and Masuda HM03, they are a far reaching generalization, beyond the spaces $X(Q, \lambda)$, of singular toric varieties having orbifold singularities. In this subsection, we recall the definition of locally standard torus orbifolds and apply Theorem 1.1 and 1.2 on this class. 
The faces of a manifold with corners can be defined following [Dav83, Section 6]. A manifold with corners is called nice if a codimension 2 face is a connected component of the intersection of two codimension 1 faces. Let $P$ be an $n$-dimensional nice manifold with corners and $\mathcal{F}(P)=\left\{F_{1}, \ldots, F_{m}\right\}$ the codimension- 1 faces of $P$. One can define $\mathcal{R}$-characteristic function $\lambda$ on $P$ satisfying the condition (4.2). In Figure 4, we give some examples of nice manifolds with corners and $\mathcal{R}$-characteristic functions on them.

A torus orbifold $X_{P}(\lambda)$ from a pair $(P, \lambda)$, where $P$ is a nice manifold with corners and $\lambda$ is an $\mathcal{R}$-characteristic function on $P$, can be defined similarly to the construction of a toric orbifold from an $\mathcal{R}$-characteristic pair, see for example KMZ, MMP07, MP06, and 4.3 in Subsection 4.1. Moreover, all the notation of the local structures, such as $g_{Q}(v)$ and $g_{E}(v)$ of a toric orbifold in Subsection 4.1 naturally extends to a torus orbifold $X_{P}(\lambda)$. We remark that a certain non-convex manifold with corners and corresponding torus manifolds are studied in PS15] .

Example 4.10. In this example we exhibit two 3-dimensional manifolds with corners and an $\mathcal{R}$-characteristic function on each. The figure (a) is a cylinder over an eye-shape, which is not a simple polytope. In figure (b), the intersection of the facets $A B C D E F$ and $B C H E F G$ is the disjoint union of edges $B C$ and $E F$, so it is also not a simple polytope.

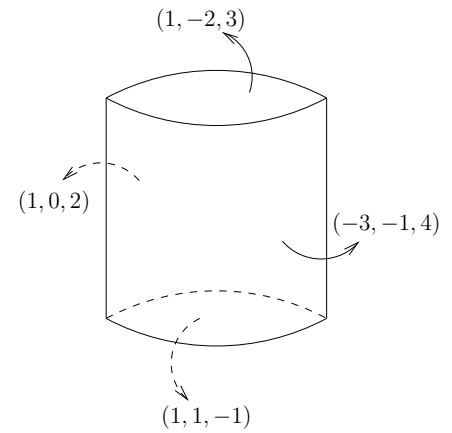

(a)

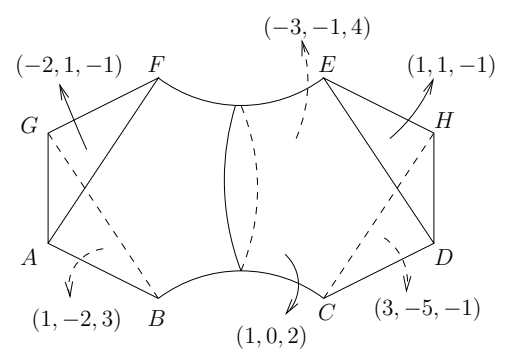

(b)

Figure 4. Two $\mathcal{R}$-characteristic functions.

We remark that a retraction sequence may exist for a nice manifold with corners which is not necessarily a simple polytope; see Figure 5 for an example. We also note that if $\left\{\left(B_{i}, E_{i}, v_{i}\right)\right\}_{i=1}^{\ell}$ is a retraction sequence of a nice manifold with corners $P$ and $X_{P}(\lambda)$ is a torus orbifold, then one can construct a building sequence for $X_{P}(\lambda)$ analogous to Proposition 4.4 . Now we can apply Theorem 1.2 to these class of orbifolds.

Corollary 4.11. Let $X_{P}(\lambda)$ be a torus orbifold over the nice manifold with corners $P$. If for each prime $p$ there is a retraction $\left\{\left(B_{i}, E_{i}, v_{i}\right)\right\}_{i=1}^{\ell}$ of $P$ such that $\operatorname{gcd}\left\{p, g_{E_{i}}\left(v_{i}\right)\right\}=1$, then $H^{*}\left(X_{P}(\lambda) ; \mathbb{Z}\right)$ is torsion free and concentrated in even degrees.

4.4. A q-cell structure for Weighted Grassmannians. In this subsection, we introduce briefly the construction of a weighted Grassmannian w $G r(d, n)$ CR02 


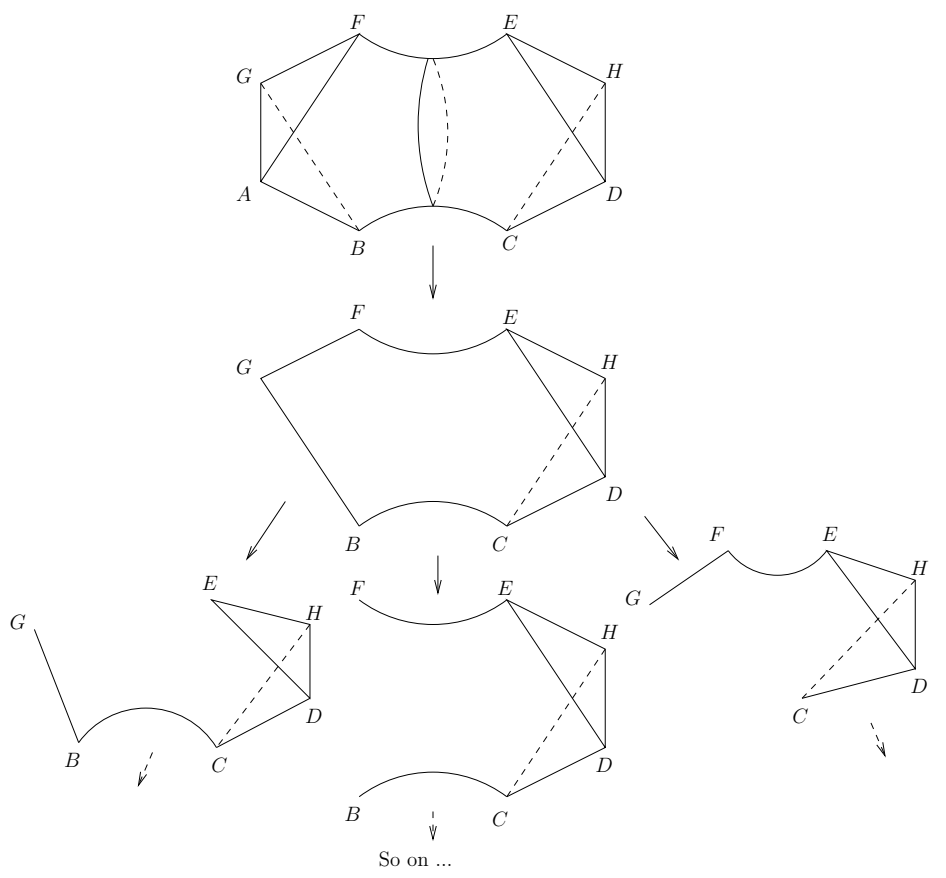

FiguRE 5. Some retraction sequences of a nice manifold with corners.

and its q-CW structure AM15, Section 2]. In addition, we show an application of Theorem 1.1 to certain weighted Grassmanians.

We first consider the action of the general linear group $G L_{n}(\mathbb{C})$ on the $d$-th exterior product $\wedge^{d} \mathbb{C}^{n}$ of $\mathbb{C}^{n}$ induced from the canonical action of $G L_{n}(\mathbb{C})$ on $\mathbb{C}^{n}$. It naturally induces the action of $G L_{n}(\mathbb{C})$ on the projective space $\mathbb{P}\left(\wedge^{d} \mathbb{C}^{n}\right)$. The standard Plücker embedding of the Grassmannian $G r(d, n)$ into $\mathbb{P}\left(\wedge^{d} \mathbb{C}^{n}\right)$ is given by the $G L_{n}(\mathbb{C})$ orbit of $\left[e_{1} \wedge \cdots \wedge e_{d}\right]$.

Now, we consider

$$
a P l(d, n)^{*}:=G L_{n}(\mathbb{C}) \cdot\left(\mathbb{C} e_{1} \wedge \cdots \wedge e_{d}\right) \backslash\{\mathbf{0}\},
$$

where $G L_{n}(\mathbb{C}) \cdot\left(\mathbb{C} e_{1} \wedge \cdots \wedge e_{d}\right)$ denotes the $G L_{n}(\mathbb{C})$-orbit of the line $\mathbb{C} e_{1} \wedge \cdots \wedge e_{d} \subset$ $\wedge^{d} \mathbb{C}^{n}$ generated by $e_{1} \wedge \cdots \wedge e_{d}$, and $\mathbf{0}$ denotes the origin in $\wedge^{d} \mathbb{C}^{n}$. Next, for the maximal torus $T$ in $G L_{n}(\mathbb{C})$, we consider

$$
K:=T \times \mathbb{C}^{*} \cong\left(\mathbb{C}^{*}\right)^{n+1}
$$

and its action on $\wedge^{d} \mathbb{C}^{n}$, where $T$ acts naturally as a subgroup of $G L_{n}(\mathbb{C})$ and $\mathbb{C}^{*}$ acts by the scalar multiplication on the vector space $\wedge^{d} \mathbb{C}^{n}$. Then, $a \operatorname{Pl}(d, n)$ is equipped with a $K$-action induced from the $K$-action on $\wedge^{d} \mathbb{C}^{n}$.

Finally, we choose a vector $\mathbf{w}:=\left(w_{1}, \ldots, w_{n}, r\right) \in \mathbb{Z}_{\geq 0}^{n} \oplus \mathbb{Z}_{>0}$ and we define

$$
\mathbf{w} D:=\left\{\left(t^{w_{1}}, \ldots, t^{w_{n}}, t^{r}\right) \in K \mid t \in \mathbb{C}^{*}\right\},
$$

the weighted diagonal subgroup of $K$ with respect to the vector w. Then, the weighted Grassmannian w $\operatorname{Gr}(d, n)$ is defined by the quotient

$$
\mathbf{w} G r(d, n):=a P l(d, n)^{*} / \mathbf{w} D .
$$


Notice that $\mathbf{w} G r(d, n)$ equips with the action of residual torus $K / \mathbf{w} D$.

Example 4.12. (1) Choose $\mathbf{w}=(0, \ldots, 0,1) \in \mathbb{Z}_{\geq 0}^{n} \oplus \mathbb{Z}_{>0}$, which defines $\mathbf{w} D=(1, \ldots, 1, t) \subset\left(\mathbb{C}^{*}\right)^{n+1}$. The quotient space $a P l(d, n)^{*} / \mathbf{w} D$ is the image of the standard Plücker embedding of Grassmannian $\operatorname{Gr}(d, n)$ into $\mathbb{P}\left(\wedge^{d} \mathbb{C}^{n}\right)$.

(2) Consider the case when $d=1$ and choose an arbitrary weighted vector $\mathbf{w}=\left(w_{1}, \ldots, w_{n}, r\right)$. Then, $G L_{n}(\mathbb{C})$-orbit of the line $\mathbb{C} e_{1}$ is isomorphic to $\mathbb{C}^{n}$. Hence, $a P l(1, n)^{*}=\mathbb{C}^{n} \backslash\{\mathbf{0}\}$. Notice that the non-zero scalar multiple of the first column of $G L_{n}(\mathbb{C})$ generates the space $\mathbb{C}^{n} \backslash\{\mathbf{0}\}$. Hence, the action of $\mathbf{w} D$ on $\mathbb{C}^{n} \backslash\{\mathbf{0}\}$ is given by

$$
\left(t^{w_{1}}, \ldots, t^{w_{n}}, t^{r}\right) \cdot\left(z_{1}, \ldots, z_{n}\right)=\left(t^{w_{1}+r} z_{1}, \ldots, t^{w_{n}+r} z_{1}\right),
$$

which leads us to the weighted projective space $\mathbb{C P}_{\left(w_{1}+r, \ldots, w_{n}+r\right)}^{n}$.

One natural way to describe a q-CW complex structure on $\mathbf{w} G r(d, n)$ is to use the classical Schubert cell decomposition of $\operatorname{Gr}(d, n)$. Let $\alpha=\left\{i_{1}<\cdots<i_{d}\right\}$ be a subset of $[n]:=\{1, \ldots, n\}$. Then, a $(d \times n)$-matrix of the following row echelon form represents a cell $e_{\alpha}$ with dimension $\sum_{k=1}^{d}\left(i_{k}-k\right)$ of $G r(d, n)$;

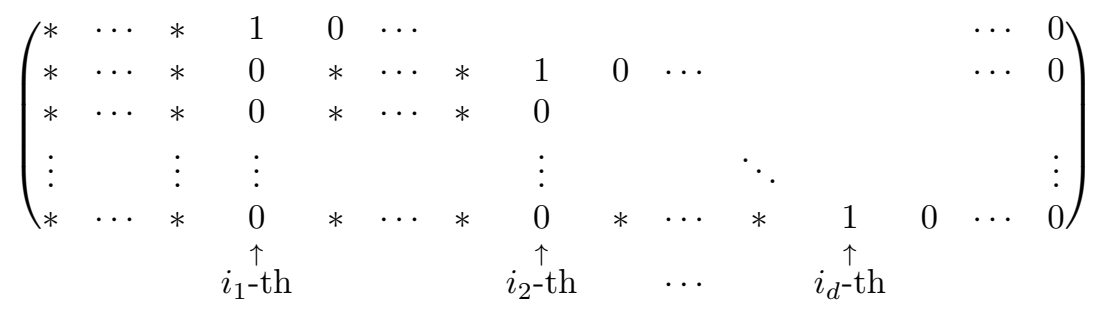

The call $e_{\alpha}$ is called the Schubert cell corresponding to the subset $\alpha$. In the literature, a Young diagram corresponding to $\left(i_{1}-1, \ldots, i_{d}-d\right)$ is used to describe $e_{\alpha}$. For example, if $n=10, d=3$ and $\alpha=\{2<5<9\}$, the Schubert cell $e_{\{2,5,9\}}$ and the corresponding Young diagram are described below in Figure 6 .

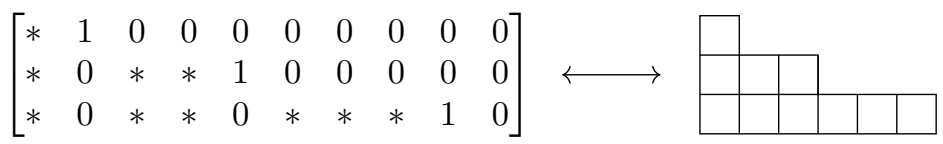

Figure 6. A Schubert cell and Young diagram.

Remark 4.13. (1) The Young diagram as defined in [Ful97] has the weakly decreasing number of boxes in each rows. In this article, we use the weakly increasing number of boxes in each rows as a convention.

(2) The collection of Young diagram has an obvious partial order, namely two Young diagrams $\square_{1} \subseteq \square_{2}$ if and only if $\square_{1}$ "fits" inside $\square_{2}$.

Finally, the Schubert cell decomposition is given by

$$
G r(d, n)=\bigsqcup_{\alpha \in[n]} e_{\alpha}
$$



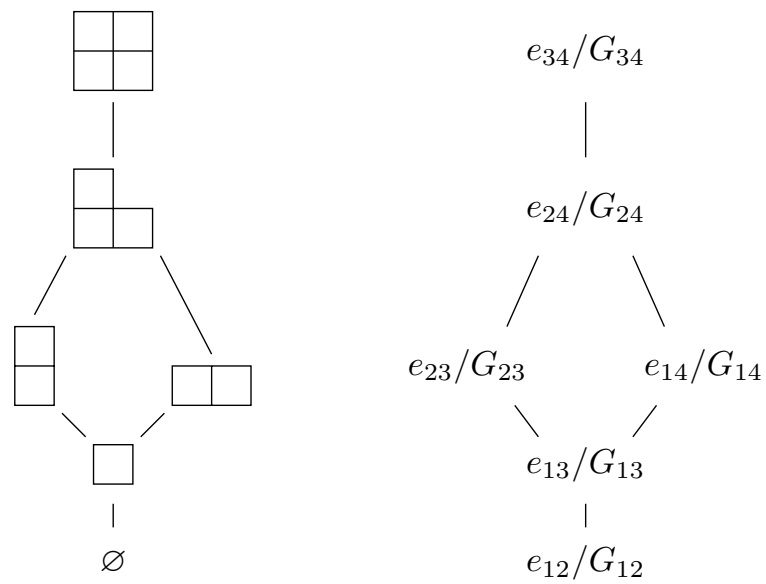

Figure 7. Lattice of Young diagrams and $\mathbf{q}-\mathrm{CW}$ structure of $\mathbf{w} G r(2,4)$.

The complex dimension of the cell $e_{\alpha}$ is exactly the number of boxes in the corresponding Young diagram, and the information about attaching maps can be obtained from the partial order given by the inclusion relations among the Young diagrams.

In order to describe a q-CW structure for $\mathbf{w} G r(d, n)$, we note that the Schubert cell decomposition (4.14 together with the orbit map $\pi: a P l(d, n)^{*} \rightarrow \operatorname{Gr}(d, n)$ induces the following cell decomposition;

$$
a P l(d, n)^{*}=\bigsqcup_{\alpha \in[n]} \pi^{-1}\left(e_{\alpha}\right) .
$$

Next, we recall from [AM15, Subsection 2.2] that each cell $\pi^{-1}\left(e_{\alpha}\right)$ is $K$-invariant, where $K$ is defined in (4.13). Hence, the cell decomposition 4.15 descends to the cell decomposition of the weighted Grassmannian.

Proposition 4.14. ([AM15, Proposition 2.3])

$$
\mathbf{w} G r(d, n)=\bigsqcup_{\alpha \in[n]} e_{\alpha} / G_{\alpha},
$$

where $G_{\alpha}=\left\{\left(t^{w_{1}}, \ldots, t^{w_{n}}, t^{r}\right) \in K \mid t \in \mathbb{C}^{*}, t^{w_{\alpha}}=1, w_{\alpha}=r+\sum_{i \in \alpha} w_{i}\right\}$.

Example 4.15. Consider the weighted Grassmannian w $G r(2,4)$ determined by the weight $\mathbf{w}=\left(w_{1}, \ldots, w_{4}, r\right) \in \mathbb{Z}_{\geq 0}^{4} \oplus \mathbb{Z}_{>0}$. The lattice structure of Young diagrams and $\mathbf{q}-\mathrm{CW}$ structure are described in Figure 7 . Here,

$$
G_{i j}=\left\{\left(t^{w_{1}}, t^{w_{2}}, t^{w_{3}}, t^{w_{4}}, t^{r}\right) \mid t \in \mathbb{C}^{*}, t^{r+w_{i}+w_{j}}=1\right\} .
$$

For example, when $\mathbf{w}=(1,1,1,1,1)$, for each $\{i, j\} \subset[4], G_{i j} \cong \mathbb{Z} / 3 \mathbb{Z}$. Hence, from Theorem 1.1. we conclude that $H^{*}(\mathbf{w} G r(2,4) ; \mathbb{Z})$ has no $p$-torsion unless $p=3$. In general, one can conclude from this observation that the cohomology ring of the weighted Grassmannian w $G r(d, n)$, determined by the weighted vector of the form $\mathbf{w}=(1, \ldots, 1,1) \in \mathbb{Z}_{\geq 0}^{n} \oplus \mathbb{Z}_{>0}$, has no $p$-torsion unless $p=d+1$. 


\section{An extension to more general q-CW complexes}

In this section, we discuss general q-CW complexes which do not necessarily consist of even dimensional q-cells. Up to this point, the degree, in the sense of Definition 2.8 has played no role. In fact, let $\left\{\left(Y_{i}, 0_{i}\right)\right\}_{i=1}^{\ell}$ be a building sequence satisfying the assumption of Theorem 1.2 . The attaching map

$$
\phi_{i}: S^{2 k_{i}-1} / G_{i} \rightarrow Y_{i-1}
$$

induces the degree for each $i=1, \ldots, \ell$. However, the induced homology map

$$
\left(\phi_{i}\right)_{*}: H_{2 k_{i}-1}\left(S^{2 k_{i}-1} / G_{i} ; \mathbb{Z}\right) \rightarrow H_{2 k_{i}-1}\left(Y_{i-1} ; \mathbb{Z}\right)
$$

is a zero map because $H_{2 k_{i}-1}\left(Y_{i-1} ; \mathbb{Z}\right)$ is trivial by Theorem 1.2 .

If a building sequence $\left\{\left(Y_{i}, 0_{i}\right)\right\}_{i=1}^{\ell}$ for a q-CW complex $X$ has odd dimensional cells, a non-trivial attaching map is possible; this leads to the following theorem.

Theorem 5.1. Let $X$ be a q- $C W$ complex, $p$ a prime number, and $\left\{\left(Y_{i}, 0_{i}\right)\right\}_{i=1}^{\ell} a$ building sequence for $X$ such that $\operatorname{gcd}\left\{p,\left|G_{i}\right|\right\}=1$ for all $i$ with $e^{k_{i}} / G_{i}=Y_{i} \backslash Y_{i-1}$. If the degree of each attaching map

$$
S^{k_{i}-1} / G_{i} \rightarrow Y_{i-1}
$$

as defined in Section 2, is coprime to $p$ or zero, then $H_{*}(X ; \mathbb{Z})$ has no p-torsion.

Proof. Since $Y_{i}$ is obtained by attaching a $\mathbf{q}$-cell $\bar{e}^{k_{i}} / G_{i}$ to $Y_{i-1}$, we have the cofibration:

$$
S^{k_{i}-1} / G_{i} \longrightarrow Y_{i-1} \longrightarrow Y_{i}
$$

which yields the long exact sequence:

$$
\cdots \rightarrow \widetilde{H}_{j}\left(S^{k_{i}-1} / G_{i}\right) \rightarrow H_{j}\left(Y_{i-1}\right) \rightarrow H_{j}\left(Y_{i}\right) \rightarrow \widetilde{H}_{j-1}\left(S^{k_{i}-1} / G_{i}\right) \rightarrow \cdots .
$$

The proof is similar to that of Theorem 1.1, except in the case when $j$ corresponds to the top two dimensions, $k_{i}$ and $k_{i}-1$. In this case, $\widetilde{H}_{k_{i}}\left(S^{k_{i}-1} / G_{i}\right)=0$ for dimensional reasons. By the same induction hypothesis as Theorem 1.1, we assume that $H_{k_{i}}\left(Y_{i-1}\right)$ and $H_{k_{i}-1}\left(Y_{i-1}\right)$ have no $p$-torsion. Hence, the sequence 5.3 becomes

$$
\begin{aligned}
& 0 \longrightarrow \mathbb{Z}^{r} \oplus K_{1} \stackrel{f}{\longrightarrow} H_{k_{i}}\left(Y_{i}\right) \stackrel{g}{\longrightarrow} \widetilde{H}_{k_{i}-1}\left(S^{k_{i}-1} / G_{i}\right) \\
& \stackrel{\left(\phi_{i}\right)_{*}}{\longrightarrow} \mathbb{Z}^{s} \oplus K_{2} \stackrel{f^{\prime}}{\longrightarrow} H_{k_{i}-1}\left(Y_{i}\right) \stackrel{g^{\prime}}{\longrightarrow}\left|G_{i}\right| \text {-torsion } \longrightarrow \cdots
\end{aligned}
$$

for some $r, s \in \mathbb{N} \cup\{0\}$ and some $p$-torsion free finite groups $K_{1}$ and $K_{2}$.

In order to show both $H_{k_{i}}\left(Y_{i}\right)$ and $H_{k_{i}-1}\left(Y_{i}\right)$ have no $p$-torsion, we need to consider the following three cases prescribed by Proposition 2.6 .

(1) If $\widetilde{H}_{k_{i}-1}\left(S^{k_{i}-1} / G_{i}\right)=0$, then $H_{k_{i}}\left(Y_{i}\right)$ is isomorphic to $\mathbb{Z}^{r} \oplus K_{1}$, hence it has no $p$-torsion. Next suppose that $H_{k_{i}-1}\left(Y_{i}\right)$ has a $p$-torsion element, say $x$, then $g^{\prime}(x)=0$ because $\operatorname{gcd}\left\{p,\left|G_{i}\right|\right\}=1$. Hence, $x \in \operatorname{ker} g^{\prime}=\operatorname{im} f^{\prime}$. Since $\widetilde{H}_{k_{i}-1}\left(S^{k_{i}-1} / G_{i}\right)=0$, the map $f^{\prime}$ is injective. It contradicts the $p$-torsion freeness of $K_{2}$.

(2) If $\widetilde{H}_{k_{i}-1}\left(S^{k_{i}-1} / G_{i}\right)$ is a non-trivial $\left|G_{i}\right|$-torsion, then an argument similar to that above shows that $H_{k_{i}}\left(Y_{i}\right)$ has no $p$-torsion. Next, consider the following exact sequence:

a $\left|G_{i}\right|$-torsion $\stackrel{\left(\phi_{i}\right)_{*}}{\longrightarrow} \mathbb{Z}^{s} \oplus K_{2} \stackrel{f^{\prime}}{\longrightarrow} H_{k_{i}-1}\left(Y_{i}\right) \stackrel{g^{\prime}}{\longrightarrow}$ a $\left|G_{i}\right|$-torsion. 
Suppose that $H_{k_{i}-1}\left(Y_{i}\right)$ has a $p$-torsion element $x$. Then the assumption $\operatorname{gcd}\left\{p,\left|G_{i}\right|\right\}=1$ implies $g^{\prime}(x)=0$. Hence, we have the following nontrivial preimage

$$
\left(f^{\prime}\right)^{-1}(x)=\left\{(a, b) \in \mathbb{Z}^{s} \oplus K_{2} \mid f^{\prime}(a, b)=x\right\} .
$$

Notice that the first coordinate of an element in 5.5 cannot be 0 , because $K_{2}$ is a $p$-torsion free finite group and for a homomorphism $h: G \rightarrow H$ of finite groups, the order of $h(a)$ divides the order of $a \in G$. Hence, there exists at least one nontrivial element $a \in \mathbb{Z}^{s}$ such that $f^{\prime}(a, b)=x$. In particular, since $x$ is a $p$-torsion element,

$$
\{n \cdot p \cdot(a, b) \mid n \in \mathbb{Z}\} \subseteq \operatorname{ker} f^{\prime}=\operatorname{im}\left(\phi_{i}\right)_{*},
$$

which contradicts the finiteness of a $\left|G_{i}\right|$-torsion.

(3) Finally, we consider the case when $\widetilde{H}_{k_{i}-1}\left(S^{k_{i}-1} / G_{i}\right)=\mathbb{Z}$ and break the exact sequence (5.4) into two parts as follows:

$$
\begin{array}{r}
0 \longrightarrow \mathbb{Z}^{r} \oplus K_{1} \stackrel{f}{\longrightarrow} H_{k_{i}}\left(Y_{i}\right) \stackrel{g}{\longrightarrow} \operatorname{im} g \longrightarrow 0, \\
0 \longrightarrow \operatorname{coker} g \stackrel{\left(\phi_{i}\right)_{*}}{\longrightarrow} \mathbb{Z}^{s} \oplus K_{2} \stackrel{f^{\prime}}{\longrightarrow} H_{k_{i}-1}\left(Y_{i}\right) \stackrel{g^{\prime}}{\longrightarrow} \mathrm{a}\left|G_{i}\right| \text {-torsion. }
\end{array}
$$

Since $\operatorname{im} g \cong n \mathbb{Z}$ for some $n \in \mathbb{N} \cup\{0\}$, applying the same argument as in Case (1) to (5.6) shows that $H_{k_{i}}\left(Y_{i}\right)$ has no $p$-torsion elements.

In order to show that $H_{k_{i}-1}\left(Y_{i}\right)$ has no $p$-torsion elements, we first assume that $g$ is a non-zero map. Then coker $g$ is finite, which implies that $H_{k_{i}-1}\left(Y_{i}\right)$ is $p$-torsion free by the same argument as in the second part of the case (2).

When the map $g$ is a zero map, then coker $g=\mathbb{Z}$. Now

$$
\left(\phi_{i}\right)_{*}: \widetilde{H}_{k_{i}-1}\left(S^{k_{i}-1} / G_{i}\right) \rightarrow H_{k_{i}-1}\left(Y_{i-1}\right)
$$

is the map induced from the attaching map $\phi_{i}: S^{k_{i}-1} / G_{i} \rightarrow Y_{i-1}$. Hence, the image of $1 \in \mathbb{Z} \cong \widetilde{H}_{k_{i}-1}\left(S^{k_{i}-1} / G_{i}\right)$ via $\left(\phi_{i}\right)_{*}$ determines the degree (see Definition 2.8) of the attaching map, which is coprime to $p$ by the assumption.

Now, we suppose that there is a $p$-torsion element $a \in H_{k_{i}-1}\left(Y_{i}\right)$. Since $g^{\prime}(a)=0$, there is an element, say $\left(\left(c_{1}, \ldots, c_{s}\right), y\right) \in \mathbb{Z}^{s} \oplus K_{2}$, such that $f^{\prime}\left(\left(c_{1}, \ldots, c_{s}\right), y\right)=a$. We notice that $\left(c_{1}, \ldots, c_{s}\right) \neq(0, \ldots, 0)$, because $f^{\prime}((0, \ldots, 0), y)$ cannot be a $p$-torsion element in $H_{k_{i}-1}\left(Y_{i}\right)$. Observe that

$$
f^{\prime}\left(p \cdot\left(c_{1}, \ldots, c_{s}\right), p \cdot y\right)=p \cdot a=0 \in H_{k_{i}-1}\left(Y_{i}\right) .
$$

Hence, there exists $m \in \mathbb{Z}$ such that

$\left(\phi_{i}\right)_{*}(m)=m \cdot\left(\phi_{i}\right)_{*}(1)=m \cdot\left(\left(d_{1}, \ldots, d_{s}\right), x\right)=\left(p \cdot\left(c_{1}, \ldots, c_{s}\right), p \cdot y\right)$.

Since the degree

$$
\operatorname{deg}\left(\phi_{i}: S^{k_{i}-1} / G_{i} \rightarrow Y_{i-1}\right)=\operatorname{gcd}\left\{d_{i} \mid 1 \leq i \leq s, d_{i} \neq 0\right\}
$$

of the attaching map is coprime to $p$ by the assumption, we conclude that $p$ divides $m$, i.e., $m=p \cdot m^{\prime}$ for some $m^{\prime} \in \mathbb{Z} \backslash\{0\}$. So, $y \equiv m^{\prime} \cdot x(\bmod p)$ and $c_{j}=m^{\prime} \cdot d_{j}$ for $j=1, \ldots, s$. Thus, we have

$a=f^{\prime}\left(\left(c_{1}, \ldots, c_{s}\right), m^{\prime} \cdot x\right)=m^{\prime} \cdot f^{\prime}\left(\left(d_{1}, \ldots, d_{s}\right), x\right)=m^{\prime} \cdot\left(f^{\prime} \circ\left(\phi_{i}\right)_{*}\right)(1)=0$ 
which is a contradiction. Hence, we conclude that $H_{k_{i}-1}\left(Y_{i}\right)$ has no $p$ torsion elements.

Corollary 5.2. If $X$ is a $\mathbf{q}-C W$ complex satisfying the assumption of Theorem 5.1 for each prime $p$, then $H_{*}(X ; \mathbb{Z})$ has no torsion.

Corollary 5.3. Let $\left\{\left(Y_{i}, 0_{i}\right)\right\}_{i=1}^{\ell}$ be a building sequence for a q-CW complex $X$ such that $H_{*}\left(Y_{i-1} ; \mathbb{Z}\right)$ has $p$-torsion and $\operatorname{gcd}\left\{p,\left|G_{j}\right|\right\}=1$ with $e^{k_{i}} / G_{i}=Y_{i} \backslash Y_{i-1}$ for $j>i$. Then $H_{*}\left(Y_{i} ; \mathbb{Z}\right)$ has p-torsion, and hence $H_{*}(X ; \mathbb{Z})$ has p-torsion.

Proof. This follows from (5.3).

\section{REFERENCES}

[AA97] Abd'Allah Al Amrani. Cohomological study of weighted projective spaces. In Algebraic geometry (Ankara, 1995), volume 193 of Lecture Notes in Pure and Appl. Math., pages 1-52. Dekker, New York, 1997.

[ALR07] Alejandro Adem, Johann Leida, and Yongbin Ruan. Orbifolds and stringy topology, volume 171 of Cambridge Tracts in Mathematics. Cambridge University Press, Cambridge, 2007.

[AM15] Hiraku Abe and Tomoo Matsumura. Equivariant cohomology of weighted Grassmannians and weighted Schubert classes. Int. Math. Res. Not. IMRN, (9):2499-2524, 2015.

[Ang13] Daniele Angella. Cohomologies of certain orbifolds. J. Geom. Phys., 71:117-126, 2013.

[Bai54] Walter L. Baily. On the quotient of an analytic manifold by a group of analytic homeomorphisms. Proc. Nat. Acad. Sci. U. S. A., 40:804-808, 1954.

[Bai56] Walter L. Baily. The decomposition theorem for $V$-manifolds. Amer. J. Math., 78:862$888,1956$.

[Bor60] Armand Borel. Seminar on transformation groups. With contributions by G. Bredon, E. E. Floyd, D. Montgomery, R. Palais. Annals of Mathematics Studies, No. 46. Princeton University Press, Princeton, N.J., 1960.

[BP02] Victor M. Buchstaber and Taras E. Panov. Torus actions and their applications in topology and combinatorics, volume 24 of University Lecture Series. American Mathematical Society, Providence, RI, 2002.

[BSS17] Anthony Bahri, Soumen Sarkar, and Jongbaek Song. On the integral cohomology ring of toric orbifolds and singular toric varieties. Algebr. Geom. Topol., 17(6):3779-3810, 2017.

[CLCT09] Tom Coates, Yuan-Pin Lee, Alessio Corti, and Hsian-Hua Tseng. The quantum orbifold cohomology of weighted projective spaces. Acta Math., 202(2):139-193, 2009.

[CLS11] David A. Cox, John B. Little, and Henry K. Schenck. Toric varieties, volume 124 of Graduate Studies in Mathematics. American Mathematical Society, Providence, RI, 2011.

[CR02] Alessio Corti and Miles Reid. Weighted Grassmannians. In Algebraic geometry, pages 141-163. de Gruyter, Berlin, 2002.

[CR04] Weimin Chen and Yongbin Ruan. A new cohomology theory of orbifold. Comm. Math. Phys., 248(1):1-31, 2004.

[Dav83] Michael Davis. Groups generated by reflections and aspherical manifolds not covered by Euclidean space. Ann. of Math. (2) 117 (1983), no. 2, 293-324.

[DJ91] Michael W. Davis and Tadeusz Januszkiewicz. Convex polytopes, Coxeter orbifolds and torus actions. Duke Math. J., 62(2):417-451, 1991.

[Ewa96] Günter Ewald. Combinatorial convexity and algebraic geometry, volume 168 of Graduate Texts in Mathematics. Springer-Verlag, New York, 1996.

[Ful93] William Fulton. Introduction to toric varieties, volume 131 of Annals of Mathematics Studies. Princeton University Press, Princeton, NJ, 1993. The William H. Roever Lectures in Geometry.

[Ful97] William Fulton. Young tableaux, volume 35 of London Mathematical Society Student Texts. Cambridge University Press, Cambridge, 1997. With applications to representation theory and geometry. 
[GM80] Mark Goresky and Robert MacPherson. Intersection homology theory. Topology, 19(2):135-162, 1980.

[Gor78] Mark Goresky. Triangulation of stratified objects. Proc. Amer. Math. Soc., 72(1):193200, 1978

[Hat02] Allen Hatcher. Algebraic topology. Cambridge University Press, Cambridge, 2002.

[HM03] Akio Hattori and Mikiya Masuda. Theory of multi-fans. Osaka J. Math., 40(1):1-68, 2003.

[Ill78] Sören Illman. Smooth equivariant triangulations of $G$-manifolds for $G$ a finite group. Math. Ann., 233(3):199-220, 1978.

[Kaw73] Tetsuro Kawasaki. Cohomology of twisted projective spaces and lens complexes. Math. Ann., 206:243-248, 1973.

[KMZ] Hideya Kuwata, Mikiya Masuda and Haozhi Zeng. Torsion in the cohomology of torus orbifolds. arXiv:1604.03138v2.

[MMP07] Hiroshi Maeda, Mikiya Masuda and Taras Panov. Torus graphs and simplicial posets. Adv. Math. 212(2):458-483, 2007.

[MP06] Mikiya Masuda and Taras Panov. On the cohomology of torus manifolds. Osaka J. Math., 43(3):711-746, 2006.

[Oda88] Tadao Oda. Convex bodies and algebraic geometry, volume 15 of Ergebnisse der Mathematik und ihrer Grenzgebiete (3) [Results in Mathematics and Related Areas (3)]. Springer-Verlag, Berlin, 1988. An introduction to the theory of toric varieties, Translated from the Japanese.

[PS10] Mainak Poddar and Soumen Sarkar. On Quasitoric Orbifolds. Osaka J. Math., 47(4): 1055-1076, 2010.

[PS15] Mainak Poddar and Soumen Sarkar. A class of torus manifolds with nonconvex orbit space. Proc. Amer. Math. Soc., 143(4):1797-1811, 2015.

[Sat56] Ichiro Satake. On a generalization of the notion of manifold. Proc. Nat. Acad. Sci. U.S.A., 42:359-363, 1956.

Department of Mathematics, Univerity of Rider, NJ, United States of America

E-mail address: bahri@rider.edu

Department of Mathematics, Vrije Universiteit Amsterdam, Amsterdam, The NetherLANDS

E-mail address: d.r.a.w.notbohm@vu.nl

Department of Mathematics, Indian Institute of Technology Madras, Chennai, India

E-mail address: soumensarkar20@gmail.com

Department of Mathematical Sciences, KAist, Daejeon, Republic of Korea

E-mail address: jongbaek.song@gmail.com 